\title{
Extended Gross-Pitaevskii description for the dynamics of thermal dilute Bose gases
}

\author{
Michiel Wouters \\ TQC, Universiteit Antwerpen, Universiteitsplein 1, B-2610 Antwerpen, Belgium
}

(Received 9 August 2013; published 10 September 2014)

\begin{abstract}
We introduce a technique to simulate the dynamics of a thermal dilute Bose gas. With the method of the nonequilibrium statistical operator, a correction to the Gross-Pitaevskii equation (GPE) is derived. This modification of the GPE describes spontaneous scattering processes. It regularizes the ultraviolet catastrophe and captures the dynamics of the particles at all energies, including the tail of the momentum distribution. We apply our model to the simulation of an evaporative cooling quench of a two-dimensional Bose gas.
\end{abstract}

DOI: 10.1103/PhysRevA.90.033611

PACS number(s): 67.85.De, 05.30.Jp

\section{INTRODUCTION}

The kinetics of the thermal weakly interacting Bose gas is a problem for which a variety of theoretical techniques have been developed [1]. In recent years, the main application has been on experiments with ultracold atomic gases [1] and quasiparticles in solid-state systems [2]. Despite the fact that no strong quantum correlations are present in the system, a truly $a b$ initio theory of the dynamics of the thermal Bose gas is still missing. This stands in stark contrast to its equilibrium properties, where Monte Carlo techniques have been very successful $[3,4]$.

The central challenge in the description of the kinetics of thermal Bose gases consists of simultaneously describing the low-energy and high-energy parts. While both behave classically, they are in the opposite classical limits of quantum mechanics: wavelike at low energy versus particlelike at high energies. This distinct behavior has led many theorists to cut phase space into a coherent region below a certain energy cutoff and an incoherent region above [see Fig. 1(a)]. The dynamics of the high-energy particles is then described by the semiclassical Boltzmann equation for particles, while the region below follows the Gross-Pitaevskii equation (GPE) for classical matter waves. The dynamics of each component being straightforward, the coupling between them is nontrivial.

For example, in the Zaremba-Nikuni-Griffin (ZNG) theory [5], a collision integral has to be evaluated to compute the scattering of high-energy particles into the low-energy region and vice versa, a calculation that always involves some approximations in practice. In the ZNG scheme, most modes are described by the Boltzmann equation, limiting the condensate to a single mode. This excludes the description of quasicondensates that appear in low dimensions [6] or in nonequilibrium turbulence [7]. In the other extreme approximation - classical field theory [8-10] — all particles are considered to be in the low-energy region and are described by the GPE. This prequantum theory consequently suffers from the ultraviolet catastrophe, that is cured by introducing a cutoff. The drawback is that physical observables become cutoff dependent. For the simulation of systems at thermal equilibrium, the cutoff dependence can be eliminated by including the particles above the cutoff in a Hartree-Fock approximation. This method however misses the dynamics of the above-cutoff particles. A similar drawback is suffered by the stochastic Gross-Pitaevskii equation (SGPE) [11-13], where the coupling between the above- and below-cutoff particles and the condensate involves an exchange of particles but does not describe the dynamics of the high-energy particles. The exception is the one-dimensional (1D) Bose gas, that does not suffer from an ultraviolet catastrophe, and the relevant physical observables [14] are cutoff independent [15-17].

The physical ingredient missing in the classical field descriptions is spontaneous scattering. Applying a randomphase approximation to the Gross-Pitaevskii equation yields a semiclassical Boltzmann equation without the spontaneous scattering terms [18]. The physical consequence is that highenergy particles have too low a probability to lose energy by scattering with other particles, leading to the slow $k^{-2}$ decay of the momentum distribution instead of the correct Maxwellian decay.

One theoretical approach that allows for the description of spontaneous scattering processes is the truncated Wigner approximation [11,19]. From the Wigner representation of the density matrix, it follows that vacuum fluctuations are described by adding half a particle per mode to the matter field. The quantum noise seeds scattering, leading to a description of spontaneous scattering events. The drawback of this method is that the quantum fluctuations tend to thermalize (reach the $k^{-2}$ momentum distribution), invalidating this approach for long-time evolutions in dimensions higher than one. A systematic expansion to beyond the truncated Wigner method was developed [20,21]. While higher-order corrections to quantum dynamics have been demonstrated for few-mode systems [22], it has not yet been applied to the long-time dynamics of continuous fields.

We adopt in this work a different approach by computing spontaneous scattering rates explicitly and adding them separately to the GPE evolution of the field, as schematically presented in Fig. 1(b). This modification of the GPE regularizes the ultraviolet catastrophe and captures the dynamics of the particles at all energies, including the tail of the momentum distribution.

In the next section, we present the model and the conditions for its validity. The derivation of the model is presented in Sec. III, and it is applied to the formation of a condensate in a two-dimensional (2D) Bose gas in Sec. IV. Conclusions are drawn in Sec. V. 


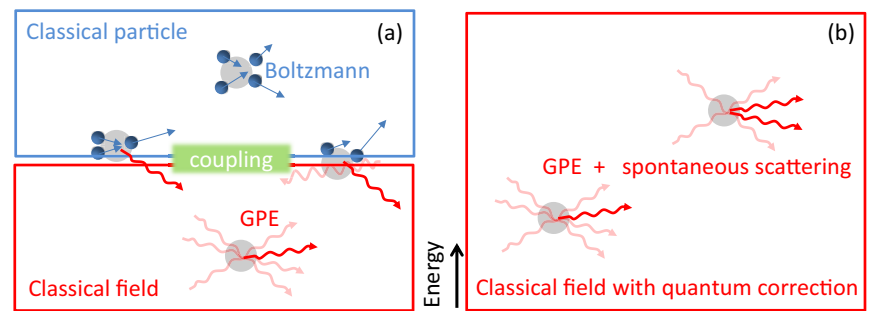

FIG. 1. (Color online) Schematic representation of (a) the standard approaches [1] to describe the thermal Bose gas vs (b) the approach adopted in this work.

\section{GPE SUPPLEMENTED WITH SPONTANEOUS SCATTERING}

For a homogenous system, our modified GPE reads explicitly in momentum space (with $\hbar=1$ )

$$
\begin{aligned}
\frac{d}{d t} \phi(\mathbf{k})= & -i\left\{\varepsilon(k) \phi(\mathbf{k})+\frac{g}{V} \sum_{k_{1}, k_{2}} \phi^{*}\left(\mathbf{k}_{3}\right) \phi\left(\mathbf{k}_{2}\right) \phi\left(\mathbf{k}_{1}\right)\right\} \\
& -R_{\mathrm{sp}, \text { out }}(\mathbf{k}) \phi(\mathbf{k})+\left.\frac{d}{d t} \phi(\mathbf{k})\right|_{\mathrm{sp}, \text { in }},
\end{aligned}
$$

where $\varepsilon(k)=k^{2} / 2 m$ ( $m$ is the boson mass) and $\mathbf{k}_{3}=\mathbf{k}_{1}+$ $\mathbf{k}_{2}-\mathbf{k}$. The evolution of the field under the in scattering leaves its phase unaltered and changes the modulus according to

$$
\frac{d}{d t}\left[|\phi(\mathbf{k})|^{2}\right]_{\mathrm{sp}, \text { in }}=R_{\mathrm{sp}, \text { in }}(\mathbf{k}) .
$$

The spontaneous in and out scattering rates are given by

$$
\begin{gathered}
R_{\mathrm{sp}, \text { in }}(\mathbf{k})=\frac{4 \pi g^{2}}{V^{2}} \sum_{\mathbf{k}_{1}, \mathbf{k}_{2}, \mathbf{k}_{3}}\left|\phi\left(\mathbf{k}_{1}\right)\right|^{2}\left|\phi\left(\mathbf{k}_{2}\right)\right|^{2} \delta\left(\Delta \epsilon_{\text {in }}\right), \\
R_{\text {sp } \text { out }}(\mathbf{k})=\frac{4 \pi g^{2}}{V^{2}} \sum_{\mathbf{k}_{1}, \mathbf{k}_{2}}\left|\phi\left(\mathbf{k}_{1}\right)\right|^{2} \delta\left(\Delta \epsilon_{\text {out }}\right),
\end{gathered}
$$

where $V$ is the system volume and the energy conservation is given by $\Delta \epsilon_{\text {in }}=\epsilon\left(\mathbf{k}_{1}\right)+\epsilon\left(\mathbf{k}_{2}\right)-\epsilon\left(\mathbf{k}_{1}+\mathbf{k}_{2}-\mathbf{k}\right)-\epsilon(\mathbf{k})$ and $\Delta \epsilon_{\text {out }}=\epsilon(\mathbf{k})+\epsilon\left(\mathbf{k}_{2}\right)-\epsilon(\mathbf{k})-\epsilon\left(\mathbf{k}+\mathbf{k}_{1}-\mathbf{k}_{2}\right)$.

The main restriction for the applicability of our method is that the spontaneous scattering rates are computed for a free particle dispersion. This is a good approximation only for the particles with kinetic energy much larger than the interaction energy, which holds for $k \gg \xi^{-1}$, where $\xi=1 / \sqrt{g n}$ is the healing length and $n$ is the density [23].

For the wave vectors in the region $\epsilon(k) \lesssim g n$, the spontaneous scattering rate is not correctly computed. Under the condition that these wave vectors enjoy large occupations $|\phi(k)|^{2} \gg 1$, the spontaneous scattering is however negligible with respect to stimulated scattering. In the numerical simulations (see below), we have found that it is actually better not to include the spontaneous scattering term when one of the final states is largely occupied. To this purpose, we have introduced a "separation momentum" $k_{s}$, that should simultaneously satisfy $\xi^{-1} \ll k_{s}$ and $\left|\phi\left(k_{s}\right)\right|^{2} \gg 1$. For a thermal Bose gas, these conditions can only be met under the condition that $g n \ll T$, a requirement that is also needed to justify the validity of standard classical field or SGPE theories. One should not confuse this separation momentum with the cutoff in those models. In the present method, the particles above $k_{s}$ are all included in the dynamics. The benefit of our approach is precisely that the low- and high-energy modes are modeled by the same equation. This results in a unified description and in particular allows for a dynamical description of the thermal cloud.

\section{DERIVATION}

Let us now turn to the derivation of Eqs. (1)-(4) with the method of the nonequilibrium statistical operator (NSO). The NSO method, developed by Zubarev et al. [24], is based on the generalization of the Gibbs ensemble to the so-called relevant ensemble. It is constructed by maximizing the entropy, keeping a set of observables fixed. Natural choices of observables in the case of the degenerate Bose gas are the field amplitude $\hat{\psi}(\mathbf{k})$ and the mode density $\hat{n}(\mathbf{k})=\hat{\psi}^{\dagger}(\mathbf{k}) \hat{\psi}(\mathbf{k})$. The relevant statistical operator for the quantum degenerate Bose gas then reads

$$
\begin{aligned}
\hat{\rho}_{\text {rel }}(t)= & \exp \left\{-\Phi(t)-\sum_{k} \xi(\mathbf{k}, t)[\hat{\psi}(\mathbf{k}, t)-\phi(\mathbf{k})]^{\dagger}\right. \\
& \times[\hat{\psi}(\mathbf{k}, t)-\phi(\mathbf{k})]\},
\end{aligned}
$$

where the Massieu-Planck function $\Phi$ serves to normalize the trace of the density matrix. The density matrix is parametrized by $\xi(\mathbf{k})$ and coherent-state amplitudes $\phi(\mathbf{k})$. The number of particles at momentum $\mathbf{k}$ is the sum of a coherent and a thermal contribution $\left\langle\hat{\psi}^{\dagger}(\mathbf{k}) \hat{\psi}(\mathbf{k})\right\rangle=|\phi(\mathbf{k})|^{2}+f_{B}[\xi(\mathbf{k})]$, where $f_{B}$ is the Bose-Einstein distribution. The density matrix (5) is not the most general Gaussian density matrix, since it is assumed that the different momentum components are independent. A treatment of quantum dynamics based on general Gaussian density matrices with applications to few mode systems is presented in Ref. [25]. For the simplicity of the numerical implementation however we will restrict it to a density matrix of the form of Eq. (5). The anomalous expectation values therefore miss the quantum fluctuations and are equal to $\langle\hat{\psi}(\mathbf{k}) \hat{\psi}(-\mathbf{k})\rangle=\phi(\mathbf{k}) \phi(-\mathbf{k})$.

In kinetic theory, equations of motion for the expectation values of operators can be derived by a perturbative expansion of the density matrix as $\hat{\rho}=\hat{\rho}_{\text {rel }}+\delta \hat{\rho}$, corresponding to a separation of the Hamiltonian in terms of free $\left(\hat{H}_{0}\right)$ and interaction $\left(\hat{H}_{I}\right)$ parts. We take as usual

$$
\hat{H}_{0}=\sum_{\mathbf{k}} \epsilon(k) \hat{\psi}^{\dagger}(\mathbf{k}) \hat{\psi}(\mathbf{k})
$$

to be the kinetic energy and

$$
\hat{H}_{I}=\frac{g}{2 V} \sum_{\mathbf{k}_{1}, \mathbf{k}_{2}, \mathbf{k}_{3}} \hat{\psi}^{\dagger}\left(\mathbf{k}_{1}+\mathbf{k}_{2}-\mathbf{k}_{3}\right) \hat{\psi}^{\dagger}\left(\mathbf{k}_{3}\right) \hat{\psi}\left(\mathbf{k}_{2}\right) \hat{\psi}\left(\mathbf{k}_{1}\right)
$$

to be the two-body interaction energy. Up to second order in the interactions, the equation of motion for the fields reads (see Ref. [24], pp. 290 and 291)

$$
\frac{d}{d t}\langle\hat{\psi}(\mathbf{k})\rangle=-i \epsilon(k)\langle\hat{\psi}(\mathbf{k})\rangle+J^{(1)}(\mathbf{k})+J^{(2)}(\mathbf{k}) .
$$

Here $J^{(1)}(\mathbf{k})=-i\left\langle\left[\hat{\psi}_{\mathbf{k}}, \hat{H}_{I}\right]\right\rangle_{\text {rel }}$ is the mean-field interaction term. Together with the first term, it yields the usual GPE for 
the Bose field. Up to first order the method of the NSO thus recovers the GPE (in the case of incoherent particles, it yields the Vlasov equation [24]).

The power of the NSO formalism is that it allows one to include higher-order corrections systematically. The secondorder collision term $J^{(2)}$ reads in Markovian approximation

$$
\begin{aligned}
J^{(2)}(\mathbf{k}, t)= & -\int_{-\infty}^{0} d t_{1} e^{\epsilon t_{1}}\left\langle\left[\hat{H}_{I}\left(t_{1}\right),\left[\hat{H}_{I}, \hat{\psi}(\mathbf{k})\right.\right.\right. \\
& \left.\left.\left.+i \sum_{\mathbf{k}} \hat{\psi}\left(\mathbf{k}^{\prime}\right) \frac{\delta J^{(1)}(\mathbf{k}, t)}{\delta\left\langle\hat{\psi}\left(\mathbf{k}^{\prime}\right)\right\rangle}\right]\right]\right\rangle_{\mathrm{rel}}
\end{aligned}
$$

where $\epsilon$ is a convergence factor that tends to zero in the end of the calculation and $\hat{H}_{I}\left(t_{1}\right)$ is the interaction Hamiltonian in the interaction picture. The second-order collision term leads in the case of an incoherent gas to the Uehling-Uhlenbeck collision integral, i.e., the semiclassical Boltzmann collision integral containing Pauli blocking factors (for fermions) or spontaneous plus stimulated scattering (for bosons).

In our case, the GPE already describes the stimulated scattering processes. The correction $J^{(2)}$ is thus expected to generate the spontaneous scattering contribution only. Working out Eq. (9) yields indeed a quantum correction to the GPE, originating from the commutator of the creation and annihilation operators. When the thermal population is set to zero $(\xi(\mathbf{k})=+\infty)$, it reads

$$
\begin{aligned}
J^{(2)}(\mathbf{k}, t)= & -\frac{g^{2}}{V^{2}} \sum_{\mathbf{k}_{1}, \mathbf{k}_{2}} \int_{-\infty}^{0} d t_{1} e^{-i \Delta \epsilon_{\text {out }} t_{1}} \\
& \times\left\langle\psi^{\dagger}\left(\mathbf{k}_{1}+\mathbf{k}_{2}-\mathbf{k}\right) \psi\left(\mathbf{k}_{2}\right) \psi\left(\mathbf{k}_{1}\right)\right\rangle_{\text {rel }} .
\end{aligned}
$$

The energy conservation factor reads $\Delta \epsilon_{\text {out }}=\epsilon(\mathbf{k})+\epsilon\left(\mathbf{k}_{1}\right)-$ $\epsilon\left(\mathbf{k}_{2}\right)-\epsilon\left(\mathbf{k}+\mathbf{k}_{1}-\mathbf{k}_{2}\right)$. The physical effect of $J^{(2)}$ is most easily seen by analyzing its effect on the number of particles at wave vector $\mathbf{k}$ :

$$
\begin{aligned}
\frac{d}{d t}\left[|\phi(\mathbf{k}, t)|^{2}\right]_{J^{(2)}} & \\
= & -\frac{g^{2}}{V^{2}} \sum_{\mathbf{k}_{1}, \mathbf{k}_{2}} \int_{-\infty}^{0} e^{-i \Delta \epsilon_{\text {out }} t_{1}} d t_{1} \\
& \times \phi^{*}(\mathbf{k}) \phi^{*}\left(\mathbf{k}_{1}+\mathbf{k}_{2}-\mathbf{k}\right) \phi\left(\mathbf{k}_{2}\right) \phi\left(\mathbf{k}_{1}\right)+\text { H.c. }
\end{aligned}
$$

If we now assume that the phases of the field at different wave vectors are random (RPA), this term describes the spontaneous scattering of particles out of the state at momentum $\mathbf{k}$ as given by $R_{\mathrm{sp}, \text { out }}$ in Eq. (1). The second-order collision term thus yields a damping of the coherent field due to out scattering. It is expected that those particles go into the thermal component. This is confirmed by a calculation of the evolution of the thermal density $n_{\text {th }}(\mathbf{k})=\left\langle\hat{\psi}^{\dagger}(\mathbf{k}) \hat{\psi}(\mathbf{k})\right\rangle-|\phi(\mathbf{k})|^{2}$ to the same accuracy as the fields:

$$
\begin{aligned}
\left.\frac{d}{d t} n_{\mathrm{th}}(\mathbf{k})\right|_{J^{(2)}}= & \frac{g^{2}}{V^{2}} \sum_{\mathbf{k}_{1}, \mathbf{k}_{2}, \mathbf{k}_{3}} \int_{-\infty}^{0} e^{-i \Delta \epsilon_{\mathrm{in}} t_{1}} d t_{1} \\
& \times \phi^{*}\left(\mathbf{k}_{1}+\mathbf{k}_{2}-\mathbf{k}_{3}\right) \phi^{*}\left(\mathbf{k}_{3}\right) \phi\left(\mathbf{k}_{2}\right) \phi\left(\mathbf{k}_{1}\right)+\text { H.c. },
\end{aligned}
$$

with $\Delta \epsilon_{\text {in }}=\epsilon\left(\mathbf{k}_{1}\right)+\epsilon\left(\mathbf{k}_{2}\right)-\epsilon\left(\mathbf{k}_{1}+\mathbf{k}_{2}-\mathbf{k}\right)-\epsilon(\mathbf{k})$. Under the random-phase assumption, this evolution equation becomes

$$
\left.\frac{d}{d t} n_{\mathrm{th}}(\mathbf{k})\right|_{J^{(2)}}=R_{\mathrm{sp}, \text { in }}(\mathbf{k}) .
$$

Including a nonzero thermal component in the dynamics of the Bose gas presents however a serious technical complication, similar to the one faced when coupling the Boltzmann equation to the GPE in the usual approaches [see Fig. 1(a)]. We therefore absorb the thermal occupation into the coherent field and set

$$
\begin{aligned}
\frac{d}{d t} n_{\mathrm{th}}(\mathbf{k}) & \rightarrow 0, \\
\frac{d}{d t}\left[|\phi(\mathbf{k})|^{2}\right] & \rightarrow \frac{d}{d t}\left[|\phi(\mathbf{k})|^{2}\right]+\left.\frac{d}{d t} n_{\mathrm{th}}(\mathbf{k})\right|_{J^{(2)}} .
\end{aligned}
$$

We then recover with Eq. (13) the term of Eq. (2) in the evolution of $\phi(\mathbf{k}, t)$. Instead of describing the high-energy modes of the Bose gas by a thermal density, we have thus used the insight from classical field theory that incoherent populations can be described by the coherent field as well, relying on the fact the GPE evolution itself randomizes the phases of the incoherent modes. Physical observables are then computed after averaging over multiple realizations of the field with random initial phases. The advantage of this approach is that there is no need for a coupling between the dynamics of the coherent amplitude $\phi(\mathbf{k})$ and an incoherent density $n_{\text {th }}$. We can use instead the full power of the GPE, that describes without significant error the scattering from the thermal cloud into the condensate region.

In the case of a one-dimensional gas, the scattering term vanishes. The evolution of the system then reduces to the classical field theory, that is known to work well in one dimension, thanks to the absence of an ultraviolet catastrophe [15-17]. The simplest nontrivial application of the present formalism is thus for the homogeneous 2D Bose gas.

\section{APPLICATION TO THE 2D BOSE GAS}

In the numerical implementation of the model Eq. (1) to the two-dimensional Bose gas, we have used a standard Monte Carlo evaluation of the spontaneous Boltzmann scattering [26]. For the evaluation of the spontaneous scattering term, the ultracold 2D Bose gas has the advantage that the scattering rate is independent of the relative velocity of the particles [27]. The total spontaneous scattering time is therefore independent of the velocity distribution and can be written as $\tau_{\text {spont }}^{-1}=$ $(g n)^{2} V=(g n)\left(V / \xi^{2}\right)$. For fixed density, this time scales proportionally to the system volume $V$ and, unfortunately, it is for large systems much larger than the chemical potential.

The probability that a pair of particles at momenta $\mathbf{k}_{1}$ and $\mathbf{k}_{2}$ scatters in the time interval $\tau_{\text {spont }}$ is proportional to $\left|\psi\left(\mathbf{k}_{1}\right)\right|^{2} \times$ $\left|\psi\left(\mathbf{k}_{2}\right)\right|^{2}$. For the Monte Carlo evaluation of the spontaneous scattering, this probability distribution is sampled by means of a random number generator. The Monte Carlo sampling introduces an additional stochastic element in the simulation of the thermal Bose gas, apart from the random phases in the initial condition. It should be clear that this stochastic 
element does not have a physical origin in the present model and could be avoided by computing the stimulated scattering by a direct summation (that would be more time consuming). It can however be expected that a more refined theory, that takes into account quantum fluctuations, would feature a similar random component due to shot noise, but this goes beyond the scope of the present treatment.

As discussed in Sec. II, the main approximation is that the spontaneous scattering is assumed to be unaffected by the modification of the quasiparticles due to interactions. This is only satisfied for energies much larger than the interaction energy $\varepsilon \gg g n$. In the opposite limit however the spontaneous scattering is negligible when $n(k) \gg 1$. Under the condition that interactions are sufficiently weak (if a temperature exists, it reads $g n \ll T$, in units where the Boltzmann constant equals one), a separation momentum $k_{s}$ exists where excitations are free-particle-like and highly occupied. Our numerical simulations have shown that artifacts are reduced by rejecting a spontaneous scattering event if the final state of at least one of the two scattering particles has a wave vector smaller than $k_{s}$.

As a specific example, we have studied a sudden quench of a quantum degenerate Bose gas as an idealized model for very rapid evaporative cooling. Evaporative cooling has been theoretically simulated with the stochastic GPE for 1D systems [28] and for three-dimensional systems to analyze the Kibble-Zurek mechanism [29] in trapped Bose gases. For simplicity, at $t=0$, all particles with a momentum higher than the "knife momentum" $k_{\mathrm{kn}}$ were taken away from a state with a small condensate fraction. Specifically, we have started from the momentum distribution $|\phi(\mathbf{k})|^{2}=n_{c} \delta(\mathbf{k})+f_{B}\left(k^{2} / 2 m-\mu\right) \Theta\left(k<k_{\mathrm{kn}}\right)$, with random phases for each field component and a condensate fraction of about $15 \%$.

The subsequent relaxation of the gas leads to a state with a larger condensate fraction of about $40 \%$ (thanks to the finite size). The growth of the condensate fraction is shown in Fig. 2(a). In panel (b), the momentum distribution at $t=350 / g n$ is shown on a semilog scale. It clearly shows a Maxwellian decay down to occupations as small as $10^{-4}$, below which deviations from the fitted Bose-Einstein distribution become apparent. The deviation is due to numerical errors in the Monte Carlo evaluation of the spontaneous scattering and can be improved by taking a shorter simulation time step. At any rate, the inclusion of the spontaneous scattering results in a strong suppression of the large momentum states with respect to the Rayleigh-Jeans distribution (red line), that is obtained with pure classical field simulations.

The low-momentum behavior can be analyzed more clearly in panel (c), where $k^{2} n(k)$ is shown on a linear scale. Again, we compare it with several analytical results. The black dashed line is the Bose-Einstein distribution of the noninteracting Bose gas. The red dashed line shows the Rayleigh-Jeans distribution of noninteracting classical waves. As it is well known from Bogoliubov theory, the momentum distribution of the interacting gas is suppressed at low $k$ (phonon region) with respect to the case of the noninteracting gas [6]. Our simulations clearly feature this suppression due to interactions. It compares indeed favorably with the analytical prediction for the thermal contribution to the degenerate Bose gas (black line) [6]:

$$
n(k)=\frac{1}{2}\left(1+\frac{\varepsilon(k)+g n_{c}}{E(k)}\right) f_{B}\left[E_{B}(k)\right]
$$

where $E_{B}=\sqrt{\varepsilon\left(\varepsilon+2 g n_{c}\right)}$ is the Bogoliubov energy, that for the $2 \mathrm{D}$ Bose gas depends in principle on the quasicondensate density $n_{\mathrm{QC}}[30,31]$. Here, we have taken $n_{\mathrm{QC}}$ to be equal to the condensate fraction, because it is clear from the first-order spatial coherence [panel (d)] that the difference between these condensate and quasicondensate densities is small due to the finite size of the simulation area. The Rayleigh-Jeans expansion of the momentum distribution of the interacting Bose gas is shown with a red line. The intersection of
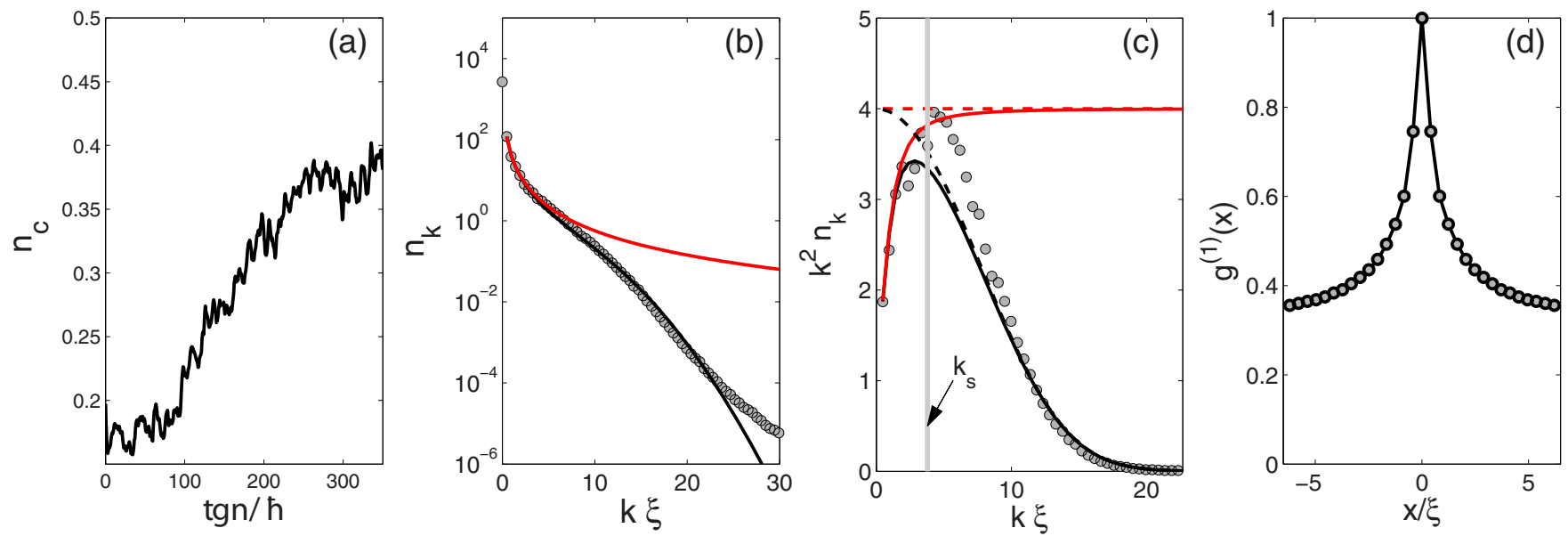

FIG. 2. (Color online) (a) Time evolution of the condensate fraction after a sudden removal of the high-energy particles with $k>k_{\mathrm{kn}}$. (b) The final momentum distribution $n(k)$ in a semilogarithmic scale. (c) $k^{2} n(k)$ in a linear scale. The separation momentum $k_{s}$ is indicated by the vertical gray line. For comparison, the analytical result (15) is shown with the black full line for a temperature $T=28 \mathrm{gn}$; the black dashed line shows the BE distribution that neglects interactions. The red full line shows the classical field limit of Eq. (15), where the red dashed line shows the classical field equipartition. (d) The first-order coherence function. System parameters: size $L_{x}=L_{y}=13 \xi$, where $\xi=\sqrt{1 /(g n m)}$ is the healing length at $T=0$. The "knife momentum" was $k_{\mathrm{kn}} \xi=9.3$ and the total number of particles was $N=7000$. The simulation was performed on a $128 \times 128$ grid, corresponding to a momentum space cutoff of $K=30 \xi^{-1}$. 

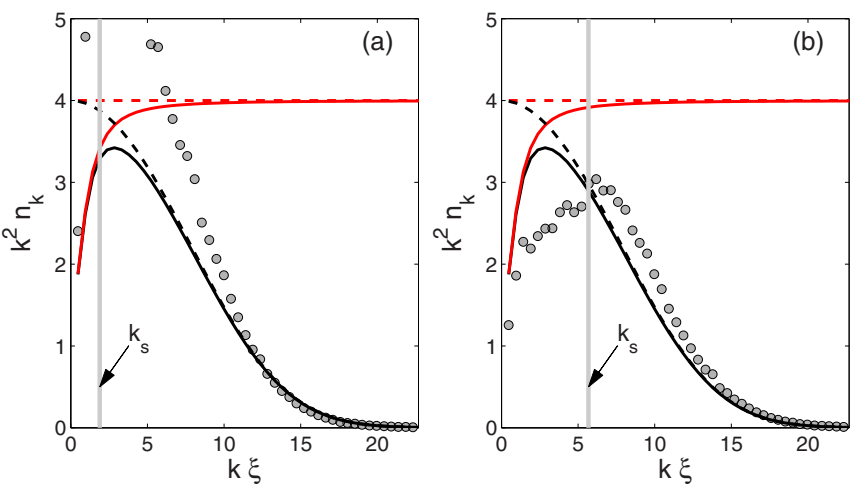

FIG. 3. (Color online) The final momentum distribution for a (a) lower and (b) higher value of the separation momentum $k_{s}$, indicated with the arrow.

the interacting classical field with the noninteracting $\mathrm{BE}$ distribution indicates where the separation momentum $k_{s}$ (indicated with a gray vertical line) should be located. In Fig. 3, we illustrate the effect of a different choice of the separation momentum. In panel (a), it is chosen too small (quadratic dispersion assumed where it is not accurate), resulting in an overestimation of the momentum distribution at small wave vectors. In panel (b) on the other hand, it is chosen too large (spontaneous scattering neglected where it is still relevant). As a consequence, the occupation of the low-momentum states is underestimated. Unfortunately, the momentum distribution depends rather sensitively on the separation momentum. An implementation of the spontaneous scattering term within the Bogoliubov framework should be able to decrease the sensitivity of the momentum distribution on $k_{s}$, but this goes beyond the scope of this work.

\section{CONCLUSIONS AND OUTLOOK}

We have derived an extension of the GPE to include spontaneous scattering processes. Our model avoids the UV catastrophe and describes the thermal cloud dynamics. It provides a unified description of the low- and high-momentum components of the Bose gas, i.e., the quasicondensate and the thermal regions.

In order to reduce artifacts of incorrectly computed spontaneous scattering rates for final states within the linear dispersion region, a separation momentum $k_{s}$ was introduced. For final states below $k_{s}$, the spontaneous scattering corrections are omitted. The error introduced in this way is small for weak interactions and sufficiently high temperatures. A possible path to improve the present model and to extend its validity to lower temperatures $T<g n$ is to extend the unperturbed Hamiltonian $H_{0}$ to the Bogoliubov one.

Further extensions of the present approach include the generalization to inhomogeneous systems. As a first step, a local-density approximation, where the spontaneous scatterings are evaluated neglecting the potential variation, seems straightforward. Applications of the formalism include experiments with ultracold atomic gases. When losses and external pumping are added to the GPE, it can also be extended to nonequilibrium polariton quantum fluids.

\section{ACKNOWLEDGMENTS}

I acknowledge Christian Maes and Wim Wagnus for pointing out the method of the nonequilibrium statistical operator and Nick Proukakis and Iacopo Carusotto for stimulating discussions on the dynamics of Bose gases. This work was financially supported by the Fonds Wetenschappelijk Onderzoek (Belgium) Odysseus program.
[1] Quantum Gases, Finite Temperature and Non-Equilibrium Dynamics, edited by N. Proukakis, S. Gardiner, M. Davis, and M. Szymańska (Imperial College Press, London, 2013).

[2] I. Carusotto and C. Ciuti, Rev. Mod. Phys. 85, 299 (2013).

[3] N. Prokof'ev, O. Ruebenacker, and B. Svistunov, Phys. Rev. Lett. 87, 270402 (2001).

[4] V. A. Kashurnikov, N. V. Prokof'ev, and B. V. Svistunov, Phys. Rev. Lett. 87, 120402 (2001).

[5] E. Zaremba, T. Nikuni, and A. Griffin, J. Low Temp. Phys. 116, 277 (1999).

[6] L. Pitaevskii and S. Stringari, Bose-Einstein Condensation (Clarendon, Oxford, 2003).

[7] N. G. Berloff and B. V. Svistunov, Phys. Rev. A 66, 013603 (2002).

[8] E. Levich and V. Yakhot, J. Phys. A 11, 2237 (1978).

[9] Yu. Kagan and B. V. Svistunov, Phys. Rev. Lett. 79, 3331 (1997).

[10] K. Góral, M. Gajda, and K. Rza̧żewski, Phys. Rev. A 66, 051602(R) (2002).

[11] P. B. Blakie, A. S. Bradley, M. J. Davis, R. J. Ballagh, and C. W. Gardiner, Adv. Phys. 57, 363 (2008).

[12] H. T. C. Stoof, J. Low Temp. Phys. 114, 11 (1999).

[13] C. W. Gardiner and M. Davis, J. Phys. B 36, 4731 (2003).
[14] The discrepancy between the $k^{-2}$ momentum tail from classical field theory and the physical Gaussian decay does not prevent good agreement between theory and experiment.

[15] M. J. Davis, P. B. Blakie, A. H. van Amerongen, N. J. van Druten, and K. V. Kheruntsyan, Phys. Rev. A 85, 031604(R) (2012).

[16] S. P. Cockburn, D. Gallucci, and N. P. Proukakis, Phys. Rev. A 84, 023613 (2011).

[17] Y. Castin, R. Dum, E. Mandonneta, A. Minguzzi, and I. Carusotto, J. Mod. Opt. 47, 2671 (2000).

[18] Y. Kagan, in Bose-Einstein Condensation, edited by S. Stringari, A. Griffin, and D. Snoke (Cambridge University, Cambridge, 1995).

[19] A. Sinatra, C. Lobo, and Y. Castin, J. Phys. B 35, 3599 (2002).

[20] A. Polkovnikov, Phys. Rev. A 68, 053604 (2003).

[21] A. Polkovnikov, Ann. Phys. 325, 1790 (2010).

[22] A. Polkovnikov, Phys. Rev. A 68, 033609 (2003).

[23] One should actually use here the quasicondensate density instead of the total density, because this is the quantity that determines the excitation spectrum [30, 31].

[24] D. Zubarev, V. Morozov, and G. Röpke, Statistical Mechanics of Nonequilibrium Processes, Volume 1: Basic Concepts, Kinetic Theory (Akademie Verlag, Berlin, 1996). 
[25] J. F. Corney and P. D. Drummond, Phys. Rev. A 68, 063822 (2003).

[26] G. A. Bird, Molecular Gas Dynamics and the Direct Simulation of Gas Flows (Clarendon, Oxford, 1994).

[27] D. S. Petrov and G. V. Shlyapnikov, Phys. Rev. A 64, 012706 (2001).
[28] B. Damski and W. H. Zurek, Phys. Rev. Lett. 104, 160404 (2010).

[29] C. N. Weiler et al., Nature (London) 455, 948 (2008).

[30] Yu. Kagan, V. A. Kashurnikov, A. V. Krasavin, N. V. Prokof'ev, and B. V. Svistunov, Phys. Rev. A 61, 043608 (2000).

[31] C. Mora and Y. Castin, Phys. Rev. A 67, 053615 (2003). 